\title{
Evolution and outcome of diastolic dysfunction
}

\author{
N Achong, S Wahi, T H Marwick
}

University of Queensland, Brisbane, Australia

Correspondence to: Professor Thomas H Marwick, University of Queensland School of Medicine, Princess Alexandra Hospital, Ipswich Road, Brisbane, Q4102, Australia; t.marwick@uq.edu.au

Accepted 24 November 2008 Published Online First 15 December 2008

\section{ABSTRACT}

Background: Diastolic dysfunction (DD) is highly prevalent and associated with increased morbidity and mortality, but its natural history remains poorly defined.

Objective: This cohort study sought to characterise the influence of clinical features, medical therapy and echocardiographic parameters on the progression of DD. Methods: We identified 926 consecutive patients laged 62 (14) years, 221 women) with DD and preserved systolic function. A repeat echocardiogram was performed in 199 patients $\geqslant 1$ year after the baseline study (average 3.6 (1.4) years). Follow-up for 4.8 (2.5) years was $97 \%$ complete for the major endpoint of all-cause mortality. Cox regression analyses were performed to identify the associations of mortality.

Results: Over follow-up, 142 patients died and 22 were admitted with heart failure. The independent predictors of death were age, hyperlipidaemia, co-morbid disease and restrictive filling. The degree of diastolic dysfunction remained stable in 52\%, deteriorated in $27 \%$ and improved in $21 \%$. There was a greater use of medical therapy in those with stable or worsening diastolic function; when the protective effects of these agents were taken into account in a multivariate model, improvement in diastolic dysfunction was associated with a survival benefit.

Conclusion: DD is associated with all-cause mortality, independent of the presence of a major co-morbidity. The degree of DD remains stable in about $50 \%$ of patients, the population whose diastolic function improves over time has a more favourable outcome.

Diastolic dysfunction (DD) is a commonly recognised echocardiographic finding, irrespective of the presence or absence of clinical features of heart failure. ${ }^{12}$ Although this abnormality may be identified regardless of the level of systolic function, it is commonly of interest in the presence of normal or near-normal systolic function. The prevalence of this problem increases with age $\mathrm{a}^{3-6}$ and is also associated with hypertension, coronary artery disease (CAD), obesity, diabetes and systolic dysfunction. ${ }^{367} \mathrm{DD}$ is believed to be at least part of the pathophysiological cause of diastolic heart failure ${ }^{8-10}$ - a condition associated with significant morbidity and mortality. ${ }^{7}{ }^{911-14}$

Three classes of DD are identified on the basis of transmitral inflow patterns. Delayed relaxation (class 1) is common with increasing age, may be associated with disturbed left ventricular (LV) loading and is associated with adverse outcome. ${ }^{3} 15$ Pseudonormal filling (class 2) and restrictive filling (class 3) are characterised by evidence of raised left atrial (LA) pressure $e^{316-18}$ and have a worse prognosis. These "stages" of diastolic dysfunction suggest that there is a passage from one stage to the next and indeed this seems plausible based on the described physiology of increasing LA pressure and decreasing LV compliance. However, the evolution of DD and its association with outcome have not been observed. In this study, we sought to determine the natural history and stability of DD using serial echocardiograms, and to determine the clinical outcomes of DD with the primary endpoint of all-cause mortality.

\section{METHODS}

\section{Study design}

We performed a cohort study derived from a consecutive group of 926 patients (62 (14) years, 221 women) undergoing echocardiograms in the years 2000 and 2001 showing DD with preserved systolic function (ejection fraction $(E F) \geqslant 45 \%$ ). Patients who had a history of valvular surgery or heart transplant were excluded. Patients were followed over 4.8 (2.5) years for death and hospital admissions, and a follow-up scan was performed in a subgroup of 199 patients after 3.6 (1.4) years (fig 1).

\section{Clinical evaluation}

Baseline data were collected regarding body-mass index (BMI), hypertension (HTN; blood pressure $\geqslant 140 / 90 \mathrm{~mm} \mathrm{Hg}$ or on treatment); hyperlipidaemia (total cholesterol $>6 \mathrm{mmol} / \mathrm{l}$ and/or LDL $>4 \mathrm{mmol} / \mathrm{l}$ or on treatment); diabetes mellitus (random blood sugar level (BSL) $>11.1 \mathrm{mmol} / \mathrm{l}$, fasting BSL $>7.0 \mathrm{mmol} / 1$ or on treatment), family history of premature coronary heart disease (CHD) (onset $<60$ years old), current or previous smoking or a history of CHD. The presence of serious comorbidities (organ failure or terminal malignancy) was noted. The clinical diagnosis of heart failure was determined using Framingham criteria. Endstage kidney disease was defined as class $\mathrm{V}$ or dialysis-dependent kidney disease. Respiratory failure was determined as type I or II respiratory failure or supplemental-oxygen dependency. Liver failure was defined as the presence of liver disease with clinical evidence of decompensation. Terminal malignancy was defined as a serious malignancy (with or without the presence of metastases) that was not amenable to curative treatment and/or treatment with palliative intent.

\section{Echocardiography}

Echocardiograms were obtained using a standard protocol and using standard commercial equipment. LA size was measured by planimetry in the apical four-chamber view. Transmitral flow was measured using pulsed-wave Doppler at the leaflet tips, aligned with the direction of LV filling. ${ }^{17}$ Diastolic dysfunction was diagnosed on the basis of increased LA size in the absence of atrial 


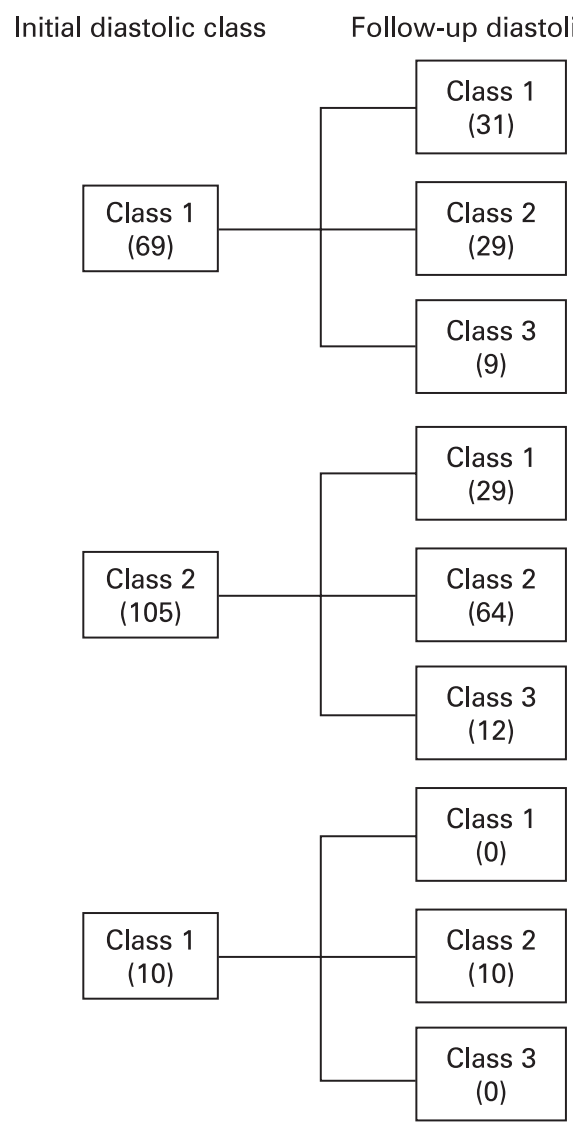

Figure 1 Progression of diastolic class in patients undergoing follow-up echocardiography.

fibrillation (AF) and mitral valve disease, with and without abnormal transmitral flow.

Of the 926 patients identified, 191 were excluded for technical reasons. Two patients had concurrent disease that could influence the assessment of diastolic function (LA mass, pericardial effusion). Seventy-nine patients had incomplete clinical data (missing or incomplete chart information). The remaining 110 patients were excluded as the $\mathrm{E}$ or $\mathrm{A}$ ratio or deceleration time could not be measured because of merging of the passive (E) and active (A) filling waves, poor imaging or other technical difficulties with the scan. In the remaining 735 patients, the class of diastolic dysfunction was determined using the age-predicted normal range for $\mathrm{E}$ wave deceleration time ${ }^{19}$ as: class Ib, delayed relaxation in the presence of LA enlargement; class II, pseudonormal filling (normal deceleration time for age in the presence of LA enlargement); and class III, restrictive filling (short deceleration time).

\section{Follow-up}

In a subgroup of 199 patients, a follow-up echocardiogram was performed at least 1 year after the initial scan (mean 3.6 (1.4) years). Of these, 15 patients were excluded on the basis of AF on the repeat echocardiogram (fig 1). Follow-up data were sought for blood pressures (obtained at outpatient visits or during hospitalisation), and correlated with changes in diastolic function. Medications were sought at the time of each echocardiogram. In order to compare doses of different agents, these were expressed as the percentage of recommended dose for each, and analysed by class. The diastolic class was assessed on the followup echocardiogram, which enabled the assessment of the change in diastolic class over time. Three groups were identified as improved, stable and deteriorated diastolic function.

Patients were followed for mortality over 4.8 (2.5) years, based on clinic attendance, chart review and standardised telephone call. The primary clinical endpoint was all-cause mortality.

\section{Statistical analysis}

An initial statistical comparison was made between the total patient population and the group lost to follow-up to determine if baseline characteristics were similar between the two populations. $\chi^{2}$ Tests were performed on categorical variables and $t$ tests or ANOVA on continuous variables. Cox regression analyses were performed to determine the univariate associations of death. The factors examined were characteristics of the patient population, cardiovascular risk factors, medications and echocardiographic parameters. Multivariate Cox models were generated to examine the independent predictors of mortality. We also sought to determine whether the stability of diastolic function over time correlated with mortality.

\section{RESULTS \\ Baseline characteristics}

The clinical status of patients at baseline is summarised in table 1 . Most patients were male, had cardiac risk factors, were taking inhibitors of the renin-aldosterone axis and over onethird had CAD. Table 1 also lists the indications for echocardiography; most tests were performed for the assessment of LV function. Most of the studies demonstrated diastolic dysfunction without other abnormalities.

\section{Clinical follow-up}

Follow-up information was obtained in 716 patients (97\%). Patients lost to follow-up had a higher prevalence of obesity, and a lower usage of nitrates, vasodilators and drugs acting against the renin-angiotensin-aldosterone axis (table 1). However, the indications and findings on echocardiography did not differ between groups (table 1). In the course of followup, there were 142 deaths.

\section{Correlates of events}

The independent correlates of mortality were age ( $\beta=1.04 ; 95 \%$ CI 1.03 to $1.06 ; p<0.001)$, hyperlipidaemia $(\beta=0.56 ; 95 \%$ CI 0.37 to $0.83 ; p=0.004)$, co-morbid disease $(\beta=10.06 ; 95 \% \mathrm{CI}$ 6.76 to $14.97 ; p<0.001)$ and restrictive filling $(\beta=2.11 ; 95 \% \mathrm{CI}$ 1.00 to $4.42 ; p=0.05)$. Other univariate associations of death that were not independent included baseline heart failure, increased body mass index) and baseline diuretic therapy.

\section{Progression of diastolic dysfunction}

Follow-up echocardiograms were performed in sinus rhythm and at least 1 year after the initial echocardiogram in 184 patients. Figure 1 illustrates the progression of diastolic class. Overall, this remained stable in 95 patients (52\%). Of the 50 patients $(27 \%)$ showing deterioration, 29 deteriorated from delayed relaxation to pseudonormal filling and nine to restrictive filling, and 12 changed from pseudonormal to restrictive filling. Of the 39 with improved function, 29 changed from pseudonormal to delayed relaxation and 10 from restrictive filling to pseudonormal. Analysis of variance demonstrated a significant association between worsening diastolic class and worsening EF ( $p=0.03)$, with a reduction of $\mathrm{EF}$ in the patients who showed a deterioration in diastolic class $(-8 \%(13 \%))$, a minor change in those with stable diastolic class 
Table 1 Clinical and echo characteristics of the total study group and those lost to follow-up

\begin{tabular}{|c|c|c|c|}
\hline & $\begin{array}{l}\text { All patients } \\
(\mathrm{n}=735)\end{array}$ & $\begin{array}{l}\text { No follow-up } \\
(\mathrm{n}=19)\end{array}$ & \\
\hline & No $(\%)$ & No (\%) & p Value \\
\hline Age (years) (SD) & $62.3(14.4)$ & $58.6(16.9)$ & 0.26 \\
\hline Male gender & $518(70.5)$ & $16(84.2)$ & 0.19 \\
\hline \multicolumn{4}{|l|}{ Co-morbidity } \\
\hline Coronary artery disease & $276(37.6)$ & $6(31.6)$ & 0.60 \\
\hline Heart failure & $2(0.3)$ & $0(0)$ & 0.82 \\
\hline Other (cancer, renal, liver, respiratory failure) & $27(3.7)$ & $0(0)$ & 0.40 \\
\hline \multicolumn{4}{|l|}{ Cardiac risk factors } \\
\hline Diabetes mellitus & $181(24.6)$ & $4(21.1)$ & 0.72 \\
\hline Hypertension & $506(68.8)$ & $12(63.2)$ & 0.60 \\
\hline Hyperlipidaemia & $363(49.4)$ & 7 (36.8) & 0.28 \\
\hline Family history & $172(23.4)$ & $7(36.8)$ & 0.17 \\
\hline Smoking & $521(70.9)$ & $11(57.9)$ & 0.22 \\
\hline Body mass index $\geqslant 30$ & $405(70.6)^{*}$ & $6(31.6)$ & 0.001 \\
\hline \multicolumn{4}{|l|}{ Medications } \\
\hline ACE/ARB/aldo antagonist & $509(69.3)$ & $7(36.8)$ & 0.003 \\
\hline$\beta$-adrenoceptor blocker & $347(47.2)$ & $9(47.4)$ & 0.69 \\
\hline Calcium channel blocker & $292(39.7)$ & $4(21.1)$ & 0.10 \\
\hline Diuretic & $264(35.9)$ & $3(15.8)$ & 0.07 \\
\hline Nitrates and vasodilators & $259(35.2)$ & $2(10.5)$ & 0.003 \\
\hline Statin, other lipid lowering & $412(56.1)$ & $9(47.4)$ & 0.23 \\
\hline \multicolumn{4}{|l|}{ Indication } \\
\hline LV function/LV hypertrophy & $325(44.2)$ & $6(31.6)$ & 0.27 \\
\hline Acute coronary syndrome & $102(13.9)$ & $2(10.5)$ & 0.68 \\
\hline Valve disease & $124(16.9)$ & $2(10.5)$ & 0.46 \\
\hline Not recorded & $184(25.0)$ & $9(47.4)$ & 0.03 \\
\hline \multicolumn{4}{|l|}{ Echo diagnosis } \\
\hline Diastolic dysfunction only & $391(53.2)$ & $7(36.8)$ & 0.16 \\
\hline Mild LV dysfunction/RWMA & $164(22.3)$ & $5(26.3)$ & 0.68 \\
\hline LV hypertrophy & $90(12.2)$ & $2(10.5)$ & 0.82 \\
\hline Valvular disease (> mild) & $64(8.7)$ & $1(5.3)$ & 0.60 \\
\hline Mild LV dysfunction & $53(7.2)$ & $2 / 19$ & 0.58 \\
\hline LA size (SD) & $26.4(4.3)$ & $26.2(3.9)$ & 0.81 \\
\hline
\end{tabular}

ACE, angiotensin-converting enzyme inhibitor; aldo, aldosterone; $A R B$, angiotensin-receptor blocker; RWMA, regional wall motion abnormality.

*Measurements were available for 574 patients.

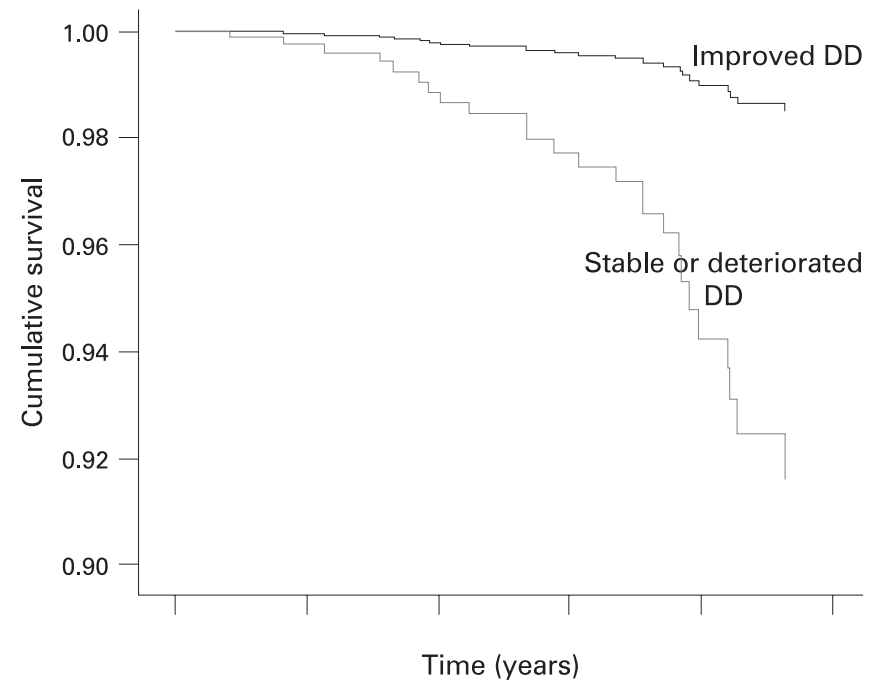

Figure 2 Survival curve comparing improved diastolic dysfunction with stable or deteriorated diastolic dysfunction (DD).
$(-1 \%(9 \%))$ and no change $(0 \%(7 \%))$ in those whose diastolic class improved.

In the subgroup of 67 patients with three or more echocardiograms performed during the study period, while the degree of diastolic dysfunction fluctuated over time, most patients showed a trend in the evolution of their DD. Twenty-two of these patients $(33 \%)$ had variable diastolic function with $77 \%$ of these fluctuating between two diastolic classes. The remaining 45 patients with three or more studies showed a linear trend in their diastolic function-13 (19\%) patients improved, 19 (28\%) patients remained stable and $13(19 \%)$ patients deteriorated. Of the patients whose DD improved, 69\% showed improvement early during the follow up period (within 2 years). However, deterioration in diastolic function occurred later in the study period (62\% deteriorating after 4 years of follow-up).

\section{Clinical correlates of alteration of diastolic function}

Deterioration of diastolic class was associated with presence of renal impairment. Improvement of diastolic class was associated with lesser requirement for medical therapy (inhibitors/antagonists of the renin-aldosterone axis and statins), probably because of a lower prevalence of hypertension. Of those that survived to have a follow-up echocardiogram, the independent correlates of outcomes in the subgroup undergoing sequential 
Table 2 Clinical correlates of the progression of diastolic class over follow-up

\begin{tabular}{|c|c|c|c|c|c|c|}
\hline & \multirow{2}{*}{$\begin{array}{l}\text { Stable }(n=95) \\
\text { No }(\%)\end{array}$} & \multicolumn{2}{|c|}{ Deteriorated $(n=50)$} & \multicolumn{3}{|c|}{ Improved $(n=39)$} \\
\hline & & No (\%) & p vs stable & No $(\%)$ & p vs stable & p vs com \\
\hline Age (years) (SD) & $62.0(12.0)$ & $61.6(12.5)$ & 0.88 & $62.1(13.8)$ & 0.96 & \\
\hline \multicolumn{7}{|l|}{ Endpoints } \\
\hline Total mortality & 17 (17.9) & $9(18)$ & 0.99 & $7(17.9)$ & 0.99 & 0.99 \\
\hline Heart failure or atrial fibrillation & $10(10.5)$ & $7(14)$ & 0.48 & $6(15.4)$ & 0.43 & 0.54 \\
\hline Coronary artery disease & $43(45.3)$ & $22(44)$ & 0.88 & $15(38.5)$ & 0.47 & 0.48 \\
\hline \multicolumn{7}{|l|}{ Risk factors } \\
\hline Diabetes mellitus & $28(29.5)$ & $16(32)$ & 0.75 & $12(30.8)$ & 0.53 & 0.96 \\
\hline Hypertension & $81(32.6)$ & $42(84)$ & 0.84 & $27(69.2)$ & 0.03 & 0.03 \\
\hline Hyperlipidaemia & $64(67.4)$ & $37(74)$ & 0.41 & $22(56.4)$ & 0.23 & 0.12 \\
\hline Family history & $17(17.9)$ & $12(24)$ & 0.38 & $8(20.5)$ & 0.72 & 0.94 \\
\hline$\beta$-blocker & $59(62.1)$ & $30(60)$ & 0.81 & $19(48.7)$ & 0.15 & 0.15 \\
\hline Calcium channel blocker & $54(56.8)$ & $26(52)$ & 0.58 & $20(51.3)$ & 0.56 & 0.67 \\
\hline Diuretic & $53(55.8)$ & $2958)$ & 0.80 & $17(43.6)$ & 0.20 & 0.15 \\
\hline Nitrate & $32(33.7)$ & $16(32)$ & 0.84 & $13(33.3)$ & 0.97 & 0.98 \\
\hline Statin & $59(62.1)$ & $30(60)$ & 0.81 & $16(41.0)$ & 0.03 & 0.02 \\
\hline
\end{tabular}

$\mathrm{ACE}$, angiotensin converting enzyme inhibitor; aldo, aldosterone; $\mathrm{ARB}$, angiotensin receptor blocker.

echocardiography were age $(\beta=1.08 ; 95 \%$ CI 1.04 to 1.13 ; $p=0.004)$, baseline heart failure $(\beta=6.28 ; 95 \%$ CI 1.33 to 29.56 ; $p=0.02)$ and LA size $(\beta=1.14 ; 95 \%$ CI 0.98 to $1.34 ; p=0.01)$, co-morbidity $(\beta=12.56 ; 95 \%$ CI 4.02 to $39.17 ; p<0.0001)$, diuretic use $(\beta=0.43 ; 95 \%$ CI 0.15 to $1.29 ; p=0.04)$ (table 2$)$. When the protective effects of these agents were taken into account in a multivariate model, improvement in diastolic dysfunction was associated with a reduction in mortality $(\beta=0.17 ; 95 \%$ CI 0.03 to $0.89 ; p=0.05$ ) (fig 2 ).

The effects of changes in blood pressure were studied in a subgroup of 74 patients who had blood pressure recorded at the time of their follow-up echocardiograms. In this subgroup, the change in BP in those improving was a reduction of $9(16) \mathrm{mm}$ $\mathrm{Hg}$, compared to an increment of $2.6(22) \mathrm{mm} \mathrm{Hg}$ in those with stable and 1 (16) $\mathrm{mm} \mathrm{Hg}$ in those with deteriorating function $(p=0.14)$. In the patients who had medication changes recorded, those with improved diastolic function had a greater increment in ACE/ARB dose than the remainder (46\% vs $18 \%$ ) but this was not significant $(p=0.22)$. Similar findings were recorded for $\beta$-blockers $(19 \%$ vs $2 \%, p=0.38)$.

\section{DISCUSSION}

The results of this cohort study confirm the association of diastolic function with all-cause mortality that has been demonstrated in previous studies. Baseline diastolic class was an independent predictor of death, irrespective of the presence of a major co-morbidity. In addition, the study shows progression of diastolic dysfunction in a substantial proportion of patients over 5 years, and demonstrates that worsening or ongoing DD is associated with mortality.

\section{Outcome of diastolic dysfunction}

Isolated LV DD is present in 35\% of hospital-based patients with CCF, who were more likely to be female and hypertensive. $^{20}$ Survival and 6-month readmission rate are similar in patients presenting with CCF attributable to systolic or diastolic dysfunction..$^{21}$ Further, increasing severity of DD correlates with a greater degree of structural abnormality and lower EF in a hospital-based setting. ${ }^{22}$ Evidence of diastolic dysfunction is also associated with reduced quality of life and was an independent risk factor for heart failure and death from cardiac causes. ${ }^{15}$ Use of diuretics alone has recently been shown to improve quality of life and symptoms of heart failure and when combined with an inhibitor of the renin-aldosterone axis, resulted in minor improvements in ventricular function. ${ }^{22}$ This study showed a trend to lower systolic BP in those who improved diastolic status, as well as greater increments in anti-hypertensive therapyalthough the latter did not reach significance.

The relation between worse levels of diastolic dysfunction and mortality replicates the findings of community-based studies. $^{3}$ Further, regardless of the class of diastolic dysfunction and when corrected for age, sex and EF, any degree of diastolic impairment was predictive of all-cause mortality. In this study, age, hyperlipidaemia and the presence of a major co-morbidity were also predictive of death.

The association between heart failure and HTN in the context of diastolic dysfunction that was demonstrated in this study is in keeping with previous studies demonstrating that an antecedent history of hypertension is common in patients presenting with diastolic heart failure. 4912132324 This relation may be due to myocardial fibrosis, which is important in hypertensive heart disease but has also been shown to correlate with severity of diastolic dysfunction. ${ }^{25}$

\section{Co-morbid illness}

Previous studies have considered the influence of selected comorbid illnesses. Redfield et $a l^{3}$ considered the influence of vascular risk factors, while Bursi et al ${ }^{26}$ found no difference in the prevalence of chronic obstructive pulmonary disease and renal impairment in patients with systolic as opposed to diastolic heart failure. These two factors were found to be independent predictors of heart failure admissions and all-cause mortality in 
a hospital-based cohort study by Berry et al. ${ }^{27}$ However, other major co-morbidities (for example, terminal malignancy or organ failure) and their potential contribution to mortality in diastolic dysfunction (not heart failure) had not previously been investigated. This is an important point, as many of these patients are elderly and the relation between worsening diastolic function and mortality could potentially have been in part attributable to other co-morbid illness. In the current study, however, we have demonstrated that although the presence of a major co-morbid illness is a predictor of mortality, diastolic class is also independently predictive of outcome.

\section{Progression of DD}

The division of diastolic dysfunction into three classes implies progression from one class to the next. However, to our knowledge, no previous studies have shown such progression to occur and the factors that influence this progression. In the current study, over 3.6 years, diastolic function remained stable in approximately half of the study population. Of the remainder, diastolic function deteriorated in $27 \%$ and improved in $21 \%$. This suggests that in about half of these patients, diastolic function does not progress with time and the division into stages or classes of severity may erroneously suggest that progression naturally occurs from one stage to the next. From the multivariate model of the predictors of all-cause mortality, progression of diastolic dysfunction, age, clinical evidence of heart failure at the time of echocardiogram, the presence of a major co-morbidity, transmitral flow and diuretic use were each independently associated with outcome. Importantly, the subgroup of patients in whom the degree of diastolic function improved with time showed a statistically significant mortality benefit compared to those whose diastolic function remained stable or deteriorated over time. This is of interest as all patients with initial class III DD who had serial echocardiograms improved to class II on follow up echocardiography. However, only a small proportion of patients (13\%) initially identified as class III had serial echocardiograms and of those whose diastolic function improved, only $25 \%$ had baseline class III DD. Further, given that the echocardiograms (at baseline and follow-up) were performed in a hospital echocardiography laboratory, it is possible that some of the change in diastolic function was attributable to changes in loading. Load dependent diastolic dysfunction has been shown to occur in normal hearts (animal and human studies) in the context of increased afterload as well as in those with heart disease despite normal haemodynamic measurements. ${ }^{28}{ }^{29}$ This may pertain, in particular, to the subgroup of patients with renal failure due to fluid retention.

\section{Limitations}

First, regarding acquisition of data, baseline echocardiograms were performed in 2000-1 when measurement of $E^{\prime}$ was not routine. Further, technical limitations of the scan necessitated the exclusion of approximately $10 \%$ of patients initially identified predominantly because mitral inflow velocities could not be measured. Patients excluded because of the presence of AF (which prohibited the analysis of transmitral flow) may also have had DD, which was not assessed. Second, information on medication use is derived from prescriptions and compliance with medications was not assessed. Similarly, BP measurements were predominantly recorded in hospital or at outpatient appointments-only a minority of patients had ambulatory 24-hour BP monitoring and therefore, the degree of control was difficult to assess.

\section{Conclusions}

$\mathrm{DD}$ is associated with all-cause mortality, which is independent of other major co-morbidities - a potential contributing factor with only limited consideration in previous studies. In a subgroup of patients we have also tracked the natural history of diastolic dysfunction which, to our knowledge, has not been done previously. The degree of diastolic function remained stable in only half of the patients, with a mortality benefit being seen in those whose diastolic function improved.

Funding: Funded in part by a project grant (456139) from the National Health and Medical Research Foundation, Canberra, Australia.

Competing interests: None.

\section{REFERENCES}

1. Gaasch WH, Zile MR. Left ventricular diastolic dysfunction and diastolic heart failure. Annu Rev Med 2004;55:373-94.

2. European Study Group on Diastolic Heart Failure. How to diagnose diastolic heart failure. Eur Heart J 1998;19:900-1003.

3. Zile MR, Brutsaert DL. New concepts in diastolic dysfunction and diastolic heart failure: part 1. Circulation 2002;105:1387-93.

4. Hogg K, Swedberg K, McMurray J. Heart failure with preserved left ventricular systolic function. Epidemiology, clinical characteristics and prognosis. J Am Col Cardiol 2004;43:317-27.

5. Zanchetti A, Cuspidi C, Comarella L, et al. Left ventricular diastolic dysfunction in elderly hypertensives; results of the APROS-diadys study. J Hyperten 2007;25:2158-67.

6. Abhayaratna WP, Marwick TH, Smith WT, et al. Characteristics of left ventricular diastolic dysfunction in the community: an echocardiographic survey. Heart 2006;92:1259-64.

7. Owan TE, Redfield MM. Epidemiology of diastolic heart failure. Prog Cardiovasc Dis 2005;47:320-32.

8. Zile MR, Bauicu CF, Gaasch VH. Diastolic heart failure-abnormalities in active relaxation and passive stiffness of the left ventricle. N Engl J Med 2004;350:777-81.

9. Senni M, Tribouilloy CM, Rodeheffer RJ, et al. Congestive heart failure in the community: a study of all incident cases in Olmsted County, Minnesota, in 1991. Circulation 1998;98:2282-9.

10. Angeja BG, Grossman W. Evaluation and management of diastolic heart failure. Circulation 2003;107:659-63.

11. Vasan RS, Larson MG, Benjamin EJ, et al. Congestive heart failure in subjects with normal versus reduced left ventricular ejection fraction: prevalence and mortality in a population-based cohort. J Am Coll Cardiol 1999;33:1948-55.

12. Kitzman DW, Gardin JM, Gottdiener JS, et al. Importance of heart failure with preserved systolic function in patients $\geqslant 65$ years of age. Am J Cardiol 2001;87:413-9.

13. Bhatia RS, Tu JV, Lee DS, et al. Outcome of heart failure with preserved ejection fraction in a population-based study. N Engl J Med 2006;355:260-9

14. Aurigemma GP, Gottdiener JS, Sheanski L, et al. Predictive value of systolic and diastolic function for incident congestive heart failure in the elderly. the Cardiovascular Health Study. J Am Coll Cardil 2001;38: 2101-13.

15. Appleton C, Hatle L, Popp R. Relationship of transmitral flow velocity patterns to left ventricular diastolic function: new insights from a combined hemodynamic and Doppler echocardiographic study. J Am Coll Cardiol 1988;12:426-40.

16. Mottram PM, Marwick TH. Assessment of diastolic function: what the general cardiologist needs to know. Heart 2005:91:681-95.

17. Leite-Moreira AF. Current perspective in diastolic dysfunction and diastolic heart failure. Heart 2006;92:712-8.

18. Klein AL, Burstow DJ, Tajik AJ, et al. Effects of age on left ventricular dimensions and filling dynamics in 117 normal persons. Mao Clin Proc 1994;69:212-24.

19. Ahmed A, Nanda N, Weaver N, et al. Clinical correlates of isolated left ventricular diastolic dysfunction among hospitalised older heart failure patients. Am J Geriatr Cardiol 2003;12:82-9.

20. Bandano LP, Albanese MC, De Biaggio P, et al. Prevalence, clinical characteristics, quality of life and prognosis of patients with congestive heart failure and isolated left ventricular diastolic dysfunction. J Am Soc Echocardiog 2004;17:253-61.

21. Yamada H, Goh PP, Sun JP, et al. Prevalence of left ventricular diastolic dysfunction by Doppler echocardiography: clinical application of the Canadian consensus guidelines. Am Soc Echocardiog 2002;15:1238-44.

22. Yip GWK, Wang M, Wang T, et al. The Hong Kong diastolic heart failure study: a randomised controlled trial of diuretics irbesartan and ramipril on quality of life, exercise capacity, left ventricular global and regional function in heart failure with a normal ejection fraction. Heart 2008;94:573-80.

23. Yamamoto K, Wilson DJ, Canzanello VJ, et al. Left ventricular diastolic dysfunction in pts with hypertension and preserved systolic function. Mayo Clin Proc 200;75:148-55.

24. Masoudi FA, Havranek EP, Smith G, et al. Gender, age and heart failure with preserved left ventricular systolic function. J Am Coll Cardiol 2003;41:217-23.

25. Martos R, Baugh J, Ledwidge $\mathrm{M}$, et al. Evidence of increased myocardial collagen turnover linked to diastolic dysfunction. Circulation 2007;115:888-95. 
26. Bursi F, Weston SA, Redfield MM, et al. Systolic and diastolic heart failure in the community. JAMA 2006;296:2209-16.

27. Berry C, Hogg K, Norrie J, et al. Heart failure with preserved left ventricular systolic function: a hospital cohort study. Heart 2005:91:907-13.
28. Gillebert TC, Leite-Moreira AF, De Hert SG. Load dependent diastolic dysfunction in heart failure. Heart Failure Rev 2000:5:345-55.

29. Leite-Moreira AF, Correia-Pinto J, Gillebert TC. Afterload induced changes in myocardial relaxation: a mechanism for diastolic dysfunction. Cardiovasc Res 1999:43:344-53.

\section{Images in cardiology}

\section{Intracoronary optical coherence tomography assessment of spontaneous coronary artery dissection}

Optical coherence tomography (OCT; ImageWire, LightLab Imaging, Westford, Massachusetts, USA) is an imaging modality with a high resolution of about 10 $20 \mu \mathrm{m}$, and can visualise the intima, media and adventitia as well as the histological examination.

A 40-year-old woman was admitted with sudden-onset chest pain at rest. She had no collagen tissue disease, no trauma, no Marfan's syndrome and Ehlers-Danlos syndrome on physical examination and no risk factors for coronary artery disease. Her admission electrocardiogram showed ST-segment depression in II, III, aVF and V4-6 leads.

Coronary angiography demonstrated an ulcer-like contrast staining (arrow head) and diffuse luminal narrowing from the distal to proximal segments in the mid left anterior descending artery (panel A). OCT examination showed the disruption of the intima (i) and media (m) (arrow), the dissection between the media $(\mathrm{m})$ and adventitia (a), and a true lumen (TL) compressed by an extensive false lumen (FL) (panels B, C and D). No atheroma was visualised in the left anterior descending artery by OCT. Two bare metal stents were directly implanted over the dissection with a good angiographic result (panel E).

We could clearly identify all layers of the vessel wall, the intimal and medial tear and the dissection between the media and adventitia by OCT. This case demonstrates that OCT can be a powerful diagnostic tool for imaging intravascular pathology in vivo.

\section{K Ishibashi, H Kitabata, T Akasaka}

kitabatanori@msn.com

Heart 2009;95:818. doi:10.1136/hrt.2008.158485
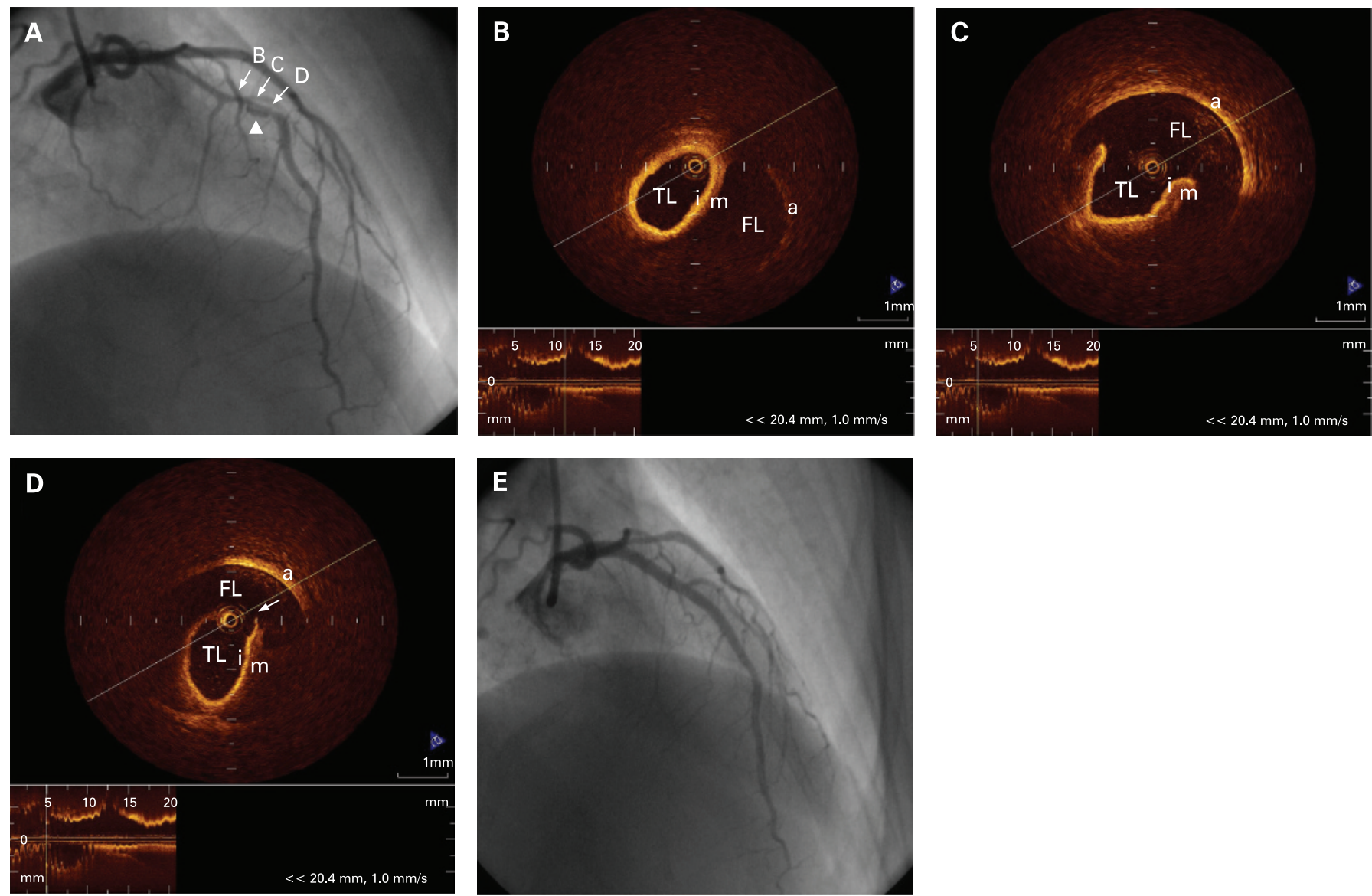